\title{
La gestion des connaissances \\ à la MRC du Domaine-du-Roy
}

\author{
Mario Gagnon ${ }^{1}$ \\ MRC du Domaine-du-Roy
}

\section{Introduction}

À l'ère de l'économie du savoir, nul n'oserait mettre en doute l'importance croissante des connaissances dans les organisations. Pourtant, bien peu d'organisations publiques peuvent, aujourd'hui, se targuer d'avoir intégré des processus de gestion des connaissances à leurs pratiques courantes de gestion ${ }^{2}$. Notre organisation, la Municipalité régionale de comté (MRC) du Domaine-duRoy, ayant récemment amorcé une démarche en ce sens, le présent article présentera l'essence de cette démarche et les premiers résultats concrets découlant de sa mise en œuvre.

\section{Mise en contexte}

Fondée il y a un peu plus de vingt ans, la MRC du Domaine-du-Roy a été, ces dernières années, comme l'ensemble des MRC au Québec, la terre d'accueil d'un grand nombre de responsabilités confiées au monde municipal. Depuis sa création, la MRC peut compter sur un directeur général et un responsable de l'aménagement du territoire. L'organisation repose en grande partie sur la compétence et le dévouement de ces personnes qui ont accumulé, au fil des ans, une foule de connaissances que l'on ne retrouve pas dans les archives. Jusqu'à maintenant, cette stabilité a permis à la MRC de retirer de nombreux bénéfices et elle a grandement facilité l'assimilation de ses nouvelles responsabilités. Une ombre plane cependant à l'horizon avec leur départ à la retraite à moyen terme. Il est donc hautement souhaitable que la MRC puisse capitaliser leurs connaissances avant qu'ils ne quittent définitivement.

\section{Définition de la gestion des connaissances}

Ce concept étant relativement nouveau pour la plupart des organisations publiques et leurs gestionnaires, j'ai reproduit une définition ${ }^{3}$ résumant bien ce qu'est la gestion des connaissances : "C'est une stratégie visant à structurer formellement le capital de connaissances explicites et tacites d'une organisation / en lien avec ses orientations stratégiques et ses besoins d'innovations et d'amélioration de compétitivité / supportée par une infrastructure technologique et organisationnelle / avec l'humain comme premier lieu d'interaction et de création de connaissance ».

Il est important de noter que la gestion des connaissances doit cibler les connaissances importantes d'une organisation. Ces connaissances doivent être en lien avec ses orientations stratégiques ou reliées aux dossiers qui revêtent une importance particulière. Il serait parfaitement utopique, fastidieux et inutile de tenter de tout capitaliser.

\section{Le concept de la gestion des connaissances}

L'objectif de cet article n'est pas de familiariser le lecteur avec le concept de la gestion des connaissances, mais il est pertinent de s'y attarder afin de bien saisir toute la portée de la gestion des savoirs. Nous verrons donc ci-après quelques extraits de deux documents traitant de ce concept, en faisant certains liens avec la démarche de la MRC.

Le premier document, «La gestion du savoir et des connaissances : apprendre en comparant les expérien- 
ces des entreprises du secteur privé et des organisations du secteur public ", émane de l'Organisation de coopération et de développement économiques (OCDE) et dresse certains constats particulièrement évocateurs relativement à la gestion du savoir et des connaissances, notamment pour les organisations publiques :

- la grande majorité des entreprises leaders du secteur privé ont aujourd'hui une politique active de gestion des connaissances, ce qui n'est pas le cas cependant pour les organisations du secteur public;

- les organisations du secteur public risquent aujourd'hui de prendre du retard par rapport aux entreprises privées précurseurs dans ce domaine, et cela risque d'avoir des conséquences néfastes pour elles;

- les instances publiques échouent régulièrement à bien maîtriser la mise en œuvre de leurs grands projets en matière de technologie de l'information.

Le premier constat traduit bien toute l'importance que les organisations devraient accorder à la gestion des connaissances. Toutefois, peu d'organisations du secteur public ont adopté des pratiques de gestion en ce sens. Il est cependant compréhensible que ces dernières soient moins enclines à investir dans une telle démarche puisque l'avantage concurrentiel qui en découle n'est pas aussi vital que pour les entreprises pri- vées. Le second constat met en lumière le risque pour les entreprises du secteur public de négliger la gestion des connaissances car, de nos jours, la main-d'œuvre est de plus en plus qualifiée et elle tend à privilégier des emplois pouvant lui offrir de meilleures perspectives de carrière par l'amélioration de ses connaissances. Les organismes publics risquent ainsi de prendre un retard qui pourrait, à long terme, les priver des meilleures ressources. Cela n'est pas sans conséquences puisque les entreprises privées produisent de plus en plus de biens et de services qui intègrent une part croissante de biens immatériels faisant directement concurrence au secteur public tels que l'éducation, la santé, la science, la sécurité et l'accès aux connaissances. Le troisième constat émet une sévère mise en garde face à la possibilité d'échec qui plane sur les grands projets impliquant l'utilisation des technologies de l'information (TI) dans le secteur public, et comme les TI sont une des composantes obligatoires de la gestion des connaissances, au même titre que les interactions humaines, cette mise en garde est importante. Ce constat nous a ainsi amenés à faire preuve de prudence dans l'utilisation des TI dans le cadre de notre démarche.

Plus loin dans ce document, on retrouve une figure intéressante (voir figure 1 que nous avons reproduite) qui illustre bien la composition du capital de connaissances d'une organisation.

\section{Figure 1 - L'essence de la connaissance}

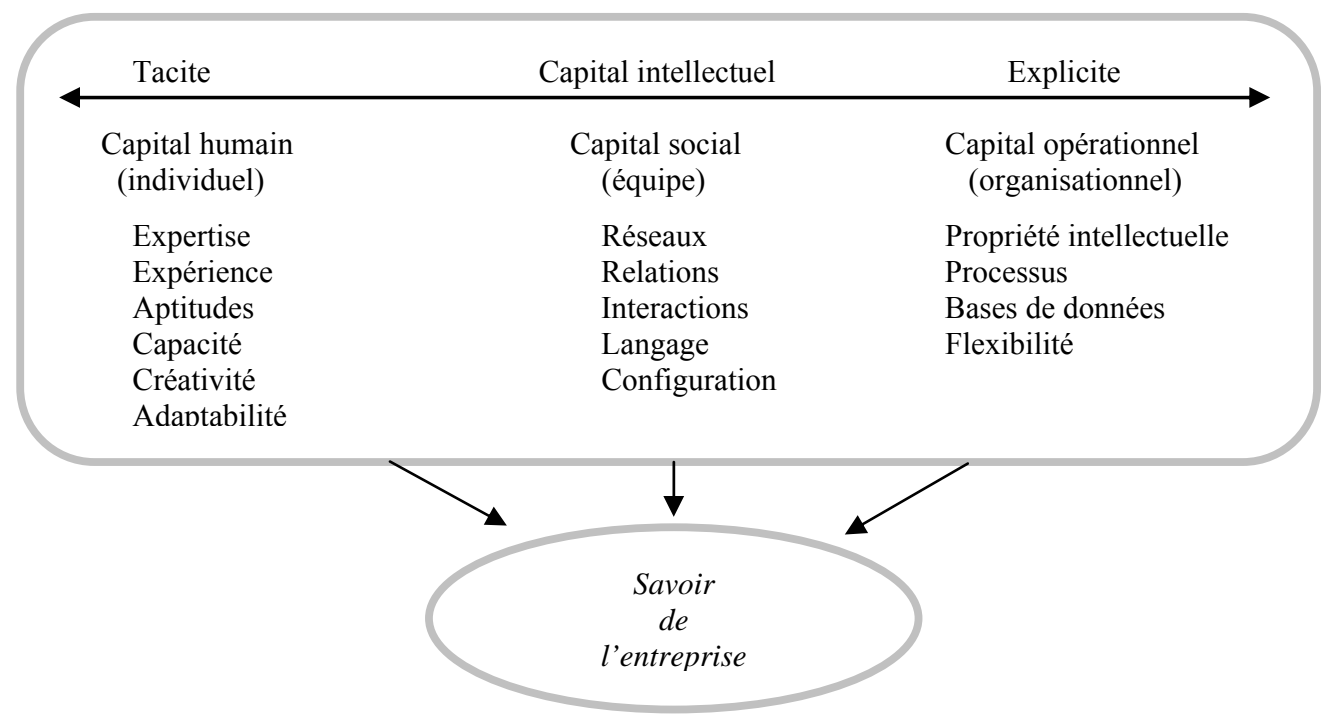


Le second document s'intitule : "Gestion des connaissances : outils et applications du Knowledge management. Dans cet ouvrage, l'auteur fait une distinction intéressante entre trois termes proches, fréquemment utilisés dans le même contexte, parfois pour désigner les mêmes choses. Pourtant, ces termes recouvrent des réalités très différentes.

\section{Donnée et information}

Une donnée est un élément brut qui n'a pas encore été mis en contexte, par exemple : $« 30^{\circ} \mathrm{C} »$. Cette donnée devient par contre une information lorsqu' on lui ajoute un contexte : « Il fait $30^{\circ} \mathrm{C}$ à Roberval ». Une information naît donc de la compréhension des relations qui peuvent exister entre les données.

\section{Connaissance}

Voyons comment cette information devient une connaissance en utilisant l'exemple précédent : «Il fera $30^{\circ} \mathrm{C}$ demain à Roberval $»$. Cette information nous permet de savoir qu'il fera chaud demain à Roberval et nous allons nous habiller en conséquence. On peut donc considérer que la connaissance devient une information comprise qui permet d'aboutir à une action.

Cet exemple illustre bien qu'une information pour une personne peut être une connaissance pour une autre en mesure de l'interpréter et de l'utiliser. Une démarche de gestion des connaissances permet cette interprétation qui donnera l'occasion à la première personne d'utiliser également cette information dans le cadre de son travail quotidien.

\section{Les formes de connaissances}

Plus loin dans le livre, on présente le modèle de Nonaka et Takeuchi qui définit deux formes de connaissances : tacite et explicite. La connaissance tacite est celle que possèdent les individus, elle n'est pas formalisée et est difficilement transmissible. Ce sont les expériences, l'intuition, les secrets du métier qu'un individu a acquis au fil des ans. C'est cependant la forme de connaissance la plus importante pour initier un processus de création de nouvelles connaissances. Elle constituerait de $85 \%$ à $90 \%$ de la connaissance d'une organisation et elle est essentiellement détenue par les individus, comme enfermée dans leur tête. La connaissance explicite est formalisée et transmissible sous forme de documents réutilisables.
Ce sont les informations concernant les dossiers, les processus, les projets, les clients, les fournisseurs, etc. En d'autres termes, ce sont les documents qui peuvent être capturés (collectés ou numérisés) et partagés par un système d'information.

\section{On peut considérer que la connaissance devient une information comprise qui permet d'aboutir à une action.}

\section{Les limites de la gestion des connaissances}

La gestion des connaissances repose sur trois facteurs essentiels : humain, organisationnel et technologique, et chacun soulève des problèmes qui ralentissent la mise en œuvre d'une démarche. En regard du facteur humain, il s'agit d'un frein énorme puisqu'il faut souvent convaincre les gens de partager leurs connaissances. À la MRC, nous n'avons cependant pas vécu cette problématique puisque le personnel cadre a accepté avec empressement de partager ses connaissances. Le problème en lien avec le facteur organisationnel émane de la structure pyramidale des organisations qui ne facilite pas l'échange des connaissances. Dans le cadre de notre démarche, compte tenu que les personnes ciblées dans l'organisation pour y participer sont directement en liens hiérarchiques, ce problème ne s'est également pas posé. Finalement, le frein lié aux technologies provient du fait que les technologies actuelles ne sont pas en mesure de gérer les connaissances tacites. Bien que ce frein puisse à lui seul décourager toute tentative d'implantation d'une démarche de gestion des connaissances, nous avons toutefois considéré qu'une capitalisation dans le cerveau d'un individu valait mieux que pas de capitalisation $\mathrm{du}$ tout, et qu'attendre le développement d'un outil technologique risquait de renvoyer notre démarche aux calendes grecques.

\section{La mise en ouvre d'une démarche}

La mise en œuvre d'une démarche peut suivre deux stratégies différentes : la stratégie de l'échange et celle de la codification. La première favorise l'échange entre les individus par la mobilisation des connaissances tacites et laisse peu de place aux documents. La seconde accorde une place importante à la codification des données; son objectif est d'assurer le partage et la transmission de l'information au sein de l'orga- 
nisation afin de la rendre disponible au plus grand nombre; en outre, elle est fortement axée sur la technologie. L'auteur mentionne toutefois qu'il est important de garder à l'esprit qu'un projet de gestion des connaissances n'est pas un projet informatique ou de gestion de l'information. À la MRC du Domaine-duRoy, nous avons retenu les deux stratégies pour l'implantation de notre démarche : la stratégie de l'échange parce que la taille réduite de notre organisation facilite les interactions humaines. Cette stratégie vise l'amélioration du capital humain (voir figure 1) en favorisant une gestion des connaissances plus individuelle, orientée vers la mobilisation des connaissances tacites (expériences, expertises, aptitudes, etc.). La codification sera également utilisée puisqu'elle permet la capitalisation de plusieurs connaissances importantes présentes sous forme de documents. Notons ici que la proximité des employés de la MRC permet de recourir à cette stratégie, malgré l'absence d'un outil technologique spécialisé pour diffuser les connaissances.

\section{Le modèle de Nonaka et Takeuchi}

La littérature en matière de gestion des connaissances révèle que Ikujiro Nonaka et Irotaka Takeuchi sont des incontournables dans ce domaine. C'est d'ailleurs à partir de leur modèle (reproduit à la figure 2) que nous avons élaboré nos processus formels de capitalisation des connaissances, comme nous le verrons d'ailleurs plus loin.

\section{Figure 2 - Modes de transfert de la connaissance}

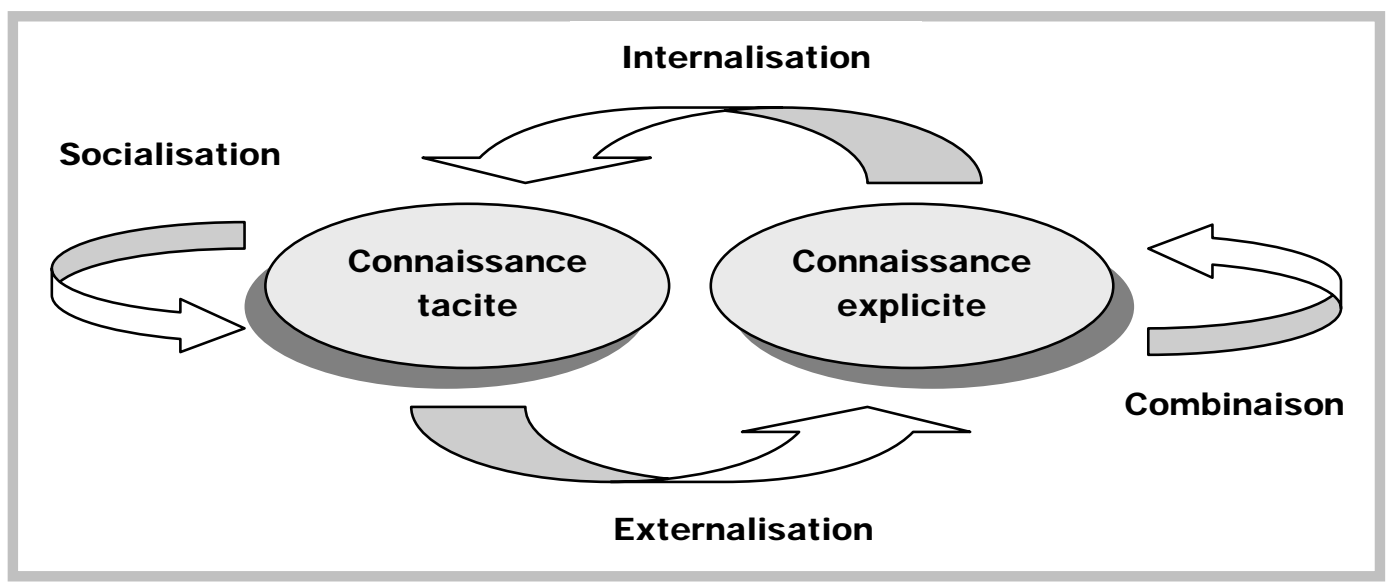

Les deux auteurs émettent l'hypothèse que la connaissance est créée à partir des différentes interactions possibles entre les connaissances tacites et explicites. Ce modèle identifie quatre modes de transfert: la socialisation, l'externalisation, l'internalisation et la combinaison.

\section{La socialisation : tacite vers tacite}

La socialisation représente le processus de transmission de connaissances tacites. Il s'agit donc de transmettre des modèles mentaux ou des compétences techniques. Cette transmission peut très bien se faire sans échanges verbaux. En effet, la transmission d'un tour de main s'effectue généralement par l'observation, l'imitation et surtout la pratique. La clé pour acquérir une connaissance tacite, c'est l'expérience.

\section{L'externalisation : tacite vers explicite}

L'externalisation est un processus qui permet le passage de connaissances tacites en connaissances explicites sous la forme de concepts, de modèles ou d'hypothèses. La modélisation d'un concept est très souvent déclenchée par le dialogue et l'échange avec d'autres individus.

\section{L’internalisation : explicite vers tacite}

L'internalisation est le processus de conversion de connaissances explicites en connaissances tacites. Typiquement, cette conversion est un processus d'apprentissage avec des supports, des documents, des manuels, etc. 
La combinaison : explicite vers explicite

La combinaison est un processus de création de connaissances explicites à partir de la restructuration d'un ensemble de connaissances explicites acquises par différents canaux de communication.

Ces quatre modes de transfert des connaissances peuvent être utilisés pour la création de nouvelles connaissances. Toutefois, pour nous simplifier la tâche, nous avons exclu l'externalisation pour amorcer notre démarche.

\section{La démarche de la MRC du Domaine-du-Roy}

La démarche de la MRC est inspirée de la chaîne de valorisation des connaissances tirée du volume de
Gilles Balmisse et que nous avons reproduite à la figure 3 .

Notre démarche a été élaborée en gardant à l'esprit que très peu d'organisations publiques ont réussi jusqu'ici à mener à bien des projets de gestion des connaissances. En ce sens, nous nous sommes limités aux trois premières étapes de la figure 3 pour l'année d'implantation. De plus, notre démarche pour la première année se concentre uniquement sur une capitalisation de haut en bas des connaissances de l'organisation, c'est-à-dire des cadres supérieurs vers leurs adjoints respectifs (les destinataires de la capitalisation) dans les deux domaines d'application les plus importants pour la MRC: la direction générale et l'aménagement du territoire. Mentionnons toutefois le potentiel fort intéressant d'éventuelles capitalisations de bas en haut et horizontales qui pourraient s'ajouter à la démarche à compter de l'an 2 .

Figure 3 - Chaîne de valorisation

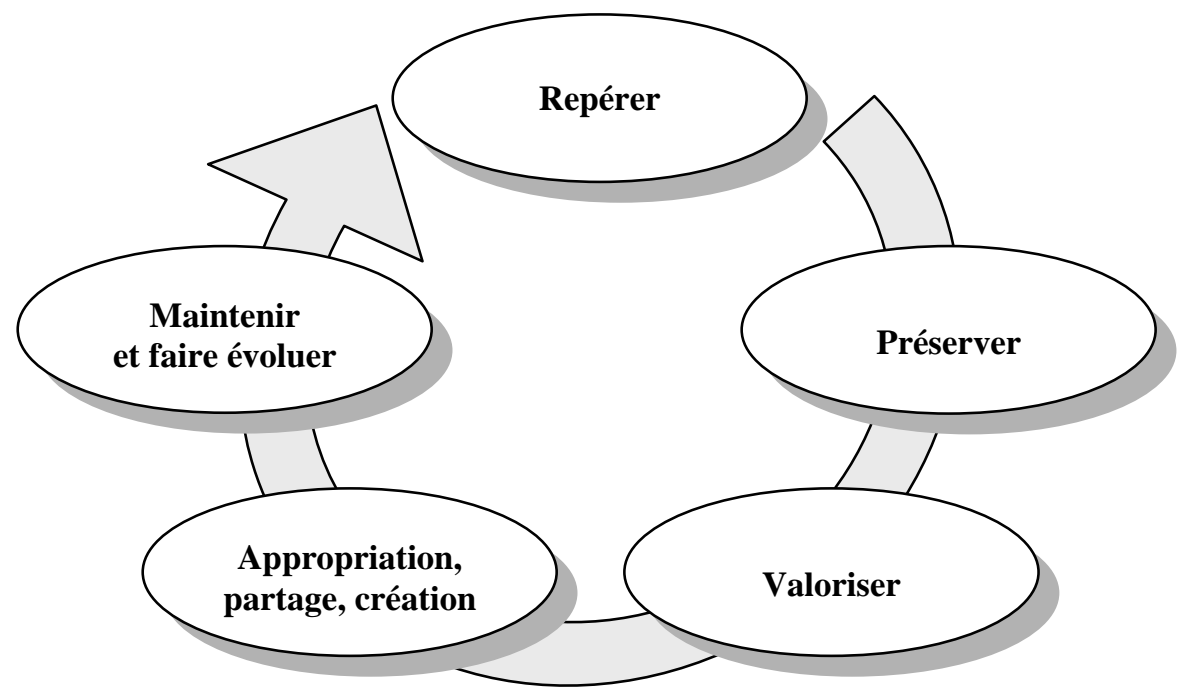

\section{Identification des connaissances à capitaliser (repérer)}

L'identification des connaissances est la première étape d'une démarche de gestion des connaissances. Il s'agit d'une étape fondamentale qui consiste à identifier, localiser et caractériser les connaissances stratégiques que l'on souhaite capitaliser, en distinguant les connaissances tacites des connaissances explicites. Pour y parvenir, nous avons d'abord dressé une liste des connaissances qui nous apparaissaient les plus susceptibles de faire l'objet de la démarche, et nous avons également passé en revue le système de gestion documentaire de la MRC. Une fois l'identification complétée, nous avons déterminé, pour chacune des connaissances retenues, le mode de transfert le plus approprié pour en effectuer la capitalisation. À la suite de cet exercice, nous avions une liste de connaissances dans chacun des domaines d'application de la démarche. 


\section{Les processus de transfert (préserver)}

Les différents processus de transfert constituent la deuxième étape d'implantation et ils sont le cœur de notre démarche. Nous présentons ici les processus de transfert que nous avons élaborés pour chacun des modes de transfert retenu.

\section{La socialisation : tacite vers tacite}

La socialisation (voir tableau 1) est le processus de transfert des connaissances tacites pour la création de nouvelles connaissances tacites, et l'échange entre deux individus constitue le meilleur moyen de créer et de partager ces connaissances. Cette capitalisation s'effectue dans le cerveau des individus.

\section{Tableau 1 - Processus de capitalisation : socialisation}

Socialisation
1. Rencontres bimestrielles distinctes, à des dates prédéterminées, entre les partici-
pants de chacun des domaines d'application.
2. Échanges, lors des rencontres, sur les connaissances difficilement transmissibles
sous forme de documents et préalablement identifiées, à savoir : les réussites, les
leçons tirées, les expériences, l'intuition, les secrets du métier, etc.
3. Délégation de tâches afin de confirmer l'acquisition et l'intégration des nouvelles
connaissances dans le quotidien des destinataires de la capitalisation.
4. Évaluation du niveau d'intégration des connaissances dans le travail quotidien des
destinataires de la capitalisation, au fil des rencontres, par les cadres supérieurs.
5. Retour sur certaines connaissances lors des rencontres bimestrielles subséquentes
(si nécessaire).

\section{L’internalisation : explicite vers tacite}

L'internalisation (voir tableau 2) est le processus de conversion de connaissances explicites en connais- sances tacites, et ce processus d'apprentissage est initié à partir de documents, de manuels, etc. Tout comme pour la socialisation, cette capitalisation s'effectue également dans le cerveau des individus.

\section{Tableau 2 - Processus de capitalisation : internalisation}

\section{Internalisation}

1. Transmission par les cadres supérieurs via Outlook (ou en main propre pour des documents non disponibles sur un support numérique) des documents préalablement identifiés aux destinataires de la capitalisation et ce, avant les rencontres bimestrielles.

2. Lecture obligatoire des documents avant les rencontres bimestrielles par les destinataires de la capitalisation.

3. Évaluation de la compréhension des documents et interprétation de ceux-ci lors des rencontres bimestrielles.

4. Délégation de tâches afin de confirmer l'acquisition et l'intégration des nouvelles connaissances dans le quotidien des destinataires de la capitalisation.

5. Évaluation du niveau d'intégration des connaissances dans le travail quotidien des destinataires de la capitalisation, au fil des rencontres, par les cadres supérieurs.

6. Retour sur certains documents lors des rencontres subséquentes (si nécessaire). 


\section{La combinaison : explicite vers explicite}

Définie comme le processus qui permet la création de connaissances explicites à partir de la restructuration d'autres connaissances explicites, la combinaison est le troisième mode de transfert utilisé dans le cadre de notre démarche. Le tableau 3 nous montre le processus de capitalisation de ce mode de transfert. Contrairement aux modes de transfert précédents, la combinaison permet une capitalisation sur un support d'information, qui demeure dans l'organisation en cas de départ.

\title{
Tableau 3 - Processus de capitalisation : combinaison
}

\begin{abstract}
Combinaison
1. Transmission par les cadres supérieurs via Outlook (ou en main propre pour des documents non disponibles sur un support numérique) des documents préalablement identifiés aux destinataires de la capitalisation et ce, avant les rencontres bimestrielles.

2. Lecture obligatoire des documents avant les rencontres bimestrielles par les destinataires de la capitalisation.
3. Évaluation de la compréhension des documents et interprétation de ceux-ci lors des rencontres bimestrielles.

4. Production par les destinataires de la capitalisation, à la suite des rencontres, d'un document synthèse démontrant la compréhension du dossier.

5. Validation du document synthèse par le directeur général ou le responsable de l'aménagement du territoire afin de s'assurer que les documents produits sont fiables et que les destinataires ont une bonne compréhension des différents dossiers traités.

6. Transmission de l'information capitalisée à la technicienne en gestion documentaire de la MRC.

7. Classement et indexation de l'information avec Logimage ${ }^{4}$, selon les différents thèmes et selon les pratiques généralement reconnues en matière de gestion documentaire.
\end{abstract}

\section{La distribution (valoriser)}

Troisième et dernière étape pour l'an 1 , la distribution vise à disséminer les connaissances explicites capitalisées à l'aide des outils informatiques. Dans notre démarche, la distribution est assurée au moyen du logiciel de gestion documentaire déjà disponible sur chacun des postes de travail. Ainsi, à partir de son poste de travail, chacun des participants à la démarche peut localiser facilement un dossier donné, le récupérer et le consulter.

\section{L'échéancier d'implantation et l'évaluation de la démarche}

Dans le but d'assurer un suivi rigoureux de la démarche, nous avons élaboré un échéancier d'implantation qui permet de bien visualiser la démarche en termes de fréquence de rencontres et de périodes de capitalisation. S'il permet difficilement de juger de la charge de travail, on peut néanmoins en déduire que nos efforts en matière de gestion des connaissances nécessitent une discipline certaine des différents acteurs impliqués.

L'échéancier comporte six rencontres de capitalisation suivies d'autant de périodes de capitalisation.

Des rencontres d'évaluation trimestrielles sont également prévues afin de revoir les modalités de fonctionnement de la démarche (si cela s'avère nécessaire), et la dernière étape consiste au dépôt d'un rapport annuel auprès du conseil de la MRC. 


\section{Nos premiers constats à la suite de l'amorce de la démarche}

Une démarche de gestion des connaissances est un exercice de longue haleine pouvant difficilement être évalué à court terme. Après les premiers mois d'implantation, nous sommes cependant en mesure de livrer nos premières constatations. À ce jour, notre échéancier d'implantation a été respecté à la lettre. Nous avons ainsi tenu trois rencontres bimestrielles de capitalisation dans chacun des domaines d'application, et chaque rencontre a fait l'objet d'un processus de capitalisation différent. Mentionnons toutefois que, même si elles n'ont lieu qu'aux deux mois, les rencontres de capitalisation arrivent vite et il faut absolument que quelqu'un de l'organisation soit responsable de la démarche afin de faire respecter l'échéancier. Nous présentons donc ci-après les résultats de la capitalisation et ce, pour chacun de nos trois processus.

\section{Le processus de socialisation (tacite vers tacite)}

Ce processus nous a fait comprendre pourquoi Jacob et Pariat comparent la difficulté d'échanger les connaissances tacites au fait d'expliquer à quelqu'un, en détail, comment s'y prendre pour nager ou faire de la bicyclette. Ainsi, pour certaines connaissances tacites telles que savoir quand et comment intervenir lors de discussions houleuses entre élus, il n'est vraiment pas évident de formuler une recette précise. Comme le mentionnait le directeur général : "Chaque situation a son contexte particulier avec des acteurs différents, et j'agis beaucoup par intuition dans ces situations ». Il a cependant souligné qu'il fallait éviter à tout prix de se mettre entre l'arbre et l'écorce en prenant parti pour l'un ou pour l'autre et suggère aussi d'essayer d'amener les élus à voir la situation sous un angle différent. Donc, pour un certain type de connaissances tacites, s'il n'y a pas de recette miracle, il y a tout de même de judicieux conseils à capitaliser.

Pour une autre connaissance que nous avons choisi de capitaliser en socialisation, soit les réseaux d'influen$c e$, la capitalisation s'est révélée très enrichissante, tout d'abord quant à l'identité des différentes personnes exerçant une influence selon les secteurs, mais aussi parce que la discussion a débordé sur la conjoncture des secteurs d'activités concernés. Ces débordements ont fourni de précieuses informations pou- vant être utilisées dans le travail quotidien du destinataire de la capitalisation, ce qui se veut le but ultime de la gestion des connaissances. À la suite des premières rencontres, le processus de socialisation s'est donc avéré très positif.

\section{Le processus de combinaison (explicite vers explicite)}

À ce stade de la démarche, le processus de combinaison est celui qui a donné les résultats les plus satisfaisants. Il exige toutefois le plus d'efforts, tout particulièrement de la part des destinataires de la capitalisation. À partir du premier sujet que nous avons capitalisé à la direction générale, soit le dossier de la délégation de la gestion des terres publiques intramunicipales (TPI) à la MRC, voici comment s'est déroulé le processus.

Ce dossier important pour la MRC remonte à 1993 et il a impliqué un grand nombre d'intervenants. Pour le profane, ce dossier est très difficile à assimiler sans une interprétation par une personne ayant vécu l'expérience depuis le début. Tel que prévu au processus, la capitalisation s'est amorcée avec la transmission de tous les documents pertinents au destinataire de la capitalisation (l'adjoint au directeur général) afin qu'il en prenne connaissance avant la rencontre de capitalisation. Notons ici qu'il est important de prévoir un laps de temps suffisant entre la transmission des documents et la rencontre d'échanges. Ce délai permet au destinataire de la capitalisation de se faire une bonne idée de l'ensemble du dossier et de noter les points nébuleux pour y trouver des éclaircissements. Toute cette préparation doit être effectuée avec soin, car elle maximise grandement l'efficience de la rencontre de capitalisation.

\section{À ce stade de la démarche, le processus de combinaison est celui qui a donné les résultats les plus satisfaisants.}

Lors de la rencontre, le destinataire de la capitalisation a livré sa compréhension du dossier au détenteur de la connaissance (le directeur général). Cette façon de procéder a permis à ce dernier de s'assurer de la bonne compréhension du dossier par le destinataire de la capitalisation, de fournir des éléments de contexte n'apparaissant pas dans les différents dossiers, mais 
qui sont parfois si importants, et d'éclaircir les points nébuleux soulevés par le destinataire. Suivant la rencontre de capitalisation, le destinataire a bénéficié de la période de capitalisation prévue à l'échéancier d'implantation pour préparer le document synthèse du dossier prévu à l'étape 4 du processus de combinaison. Le document synthèse a ensuite été transmis au détenteur de la connaissance pour validation (étape 5). Cette validation nous a permis de nous assurer de la fiabilité du document et d'attester que le destinataire de la capitalisation possédait bien le dossier traité. À la lecture de la synthèse produite, le directeur général a apporté certaines précisions au document afin que l'information à capitaliser soit la plus complète possible. À la suite de cette étape, nous avions entre les mains un document d'une dizaine de pages qui intégrait les savoirs du détenteur de la connaissance et une quantité appréciable de documents accumulés au fil des onze dernières années. Ce document fut par la suite transmis à la technicienne en documentation pour compléter les deux dernières étapes du processus de combinaison.

Comme nous l'avons dit précédemment, le processus de combinaison est assez exigeant, mais les efforts consentis nous ont apporté énormément de satisfaction en regard de ce dossier important pour l'organisation. De plus, cela nous a démontré hors de tout doute l'utilité et la pertinence d'investir un peu de temps dans la gestion de nos connaissances.

\section{La volonté des acteurs de la démarche de partager leurs connaissances et d'y consacrer temps et efforts s'avère l'élément fondamental de la réussite de toute démarche de gestion des connaissances.}

\section{Le processus d'internalisation (explicite vers tacite)}

Ce processus s'avérant très semblable (les trois premières étapes sont identiques) au processus de combinaison, nous n'en ferons pas ici une description aussi exhaustive. Il est toutefois important de mettre en relief que la capitalisation ne s'effectue pas sur un support d'information, mais dans le cerveau des individus. Par ailleurs, tout comme pour le processus de combinaison, la rencontre de capitalisation doit être préparée avec attention par le destinataire de la capitalisation.

À une époque où les regroupements de structures municipales sont d'actualité, nous avons choisi de capitaliser en internalisation tout le contexte entourant la création de la MRC en 1983. À cette époque, ce dossier avait fait couler beaucoup d'encre et suscité bien des passions quant à la présence d'une ou de deux MRC sur le territoire du comté Roberval. Lors de la rencontre de capitalisation, nous avons encore une fois constaté que l'interprétation du dossier par une personne ayant vécu l'expérience en facilite grandement la compréhension. Cette interprétation permet la capitalisation de plusieurs informations et éléments de contexte ne figurant nulle part dans les différents documents et qui auraient très probablement été perdus pour l'organisation dans un proche avenir. À l'instar des processus précédents, l'internalisation a donc également contribuée à une capitalisation des connaissances de l'organisation.

\section{Conclusion}

La démarche que nous avons élaborée repose sur des fondements théoriques solides en tirant profit des avantages que procure une organisation telle que la MRC du Domaine-du-Roy. Bien que les différents processus de transfert que nous ayons élaborés soient somme toute relativement simples, il n'en demeure pas moins que l'implantation d'une démarche de gestion des connaissances est un défi pour une organisation publique. En ce sens, la volonté des acteurs de la démarche de partager leurs connaissances et d'y consacrer temps et efforts s'avère, selon nous, l'élément fondamental de la réussite de toute démarche de gestion des connaissances.

Dans le contexte actuel au Québec où nous serons bientôt confrontés au départ à la retraite d'une génération importante de travailleurs détenant une masse considérable de connaissances, la gestion de ces connaissances prend tout son sens. En effet, les savoirs et savoir-faire risquent de s'envoler des organisations avec le départ des baby-boomers, ce qui pourrait avoir de lourdes conséquences sociales si nous considérons la prépondérance de nos services publics dans notre vie quotidienne. Malgré cette évidence, bien peu d'organisations publiques ont présentement intégré une démarche formelle de gestion des connaissances à 
leurs pratiques courantes de gestion. À la lumière des premiers résultats que nous avons obtenus avec notre démarche, nous pouvons aujourd'hui témoigner que les organisations publiques auraient grandement avantage à imiter les entreprises leaders du secteur privé en se préoccupant dès à présent de la préservation de leurs connaissances.

\section{Notes et références}

1 Mario Gagnon est directeur général adjoint à la MRC du Domaine-du-Roy.
2 Organisation de coopération et de développement économiques (2001). La gestion du savoir et des connaissances : apprendre en comparant les expériences des entreprises du secteur privé et des organisations du secteur public, compte rendu succinct du forum de haut niveau organisé à Copenhague, les 8 et 9 février 2001, Bibliothèque de l'ÉNAP.

3 Jacob, Réal et Lucile Pariat (2002). Savez-vous vraiment ce que vous savez ? RÉSEAU CEFRIO, vol. $3 \mathrm{n}^{\circ}$ 2, mars.

$4 \quad$ Logimage est le logiciel de gestion documentaire utilisé à la MRC du Domaine-du-Roy depuis quelques années. 